\title{
Performance Assessment of BDS and GPS/BDS Velocity Estimation with Stand-alone Receiver
}

\author{
Kai Zheng and Long Tang \\ (Wuhan University, P.R. China) \\ (E-mail: 1tang@whu.edu.cn)
}

\begin{abstract}
Accurate velocity estimates are critical in highly dynamic positioning, airborne gravimetry, and geophysics applications. This paper focuses on the evaluation of the performance of velocity estimation using the BeiDou navigation satellite system (BDS) alone and integrated Global Positioning System (GPS)/BDS. Firstly, we analyse and compare the position-derivation method and analytical method which are used to calculate BDS satellite velocity from broadcast ephemeris. Results show that the accuracy of the estimated velocity by position-derivation method can be within $1 \mathrm{~mm} / \mathrm{s}$ and better than that of the analytical method. Secondly, velocity estimation tests were carried out both in static and kinematic modes. The results show that: 1) the accuracy of BDS velocity estimation is in the same order of magnitude to that of GPS; 2) Compared with a single navigation system, the stability and accuracy of velocity estimation can be remarkably improved by integrated GPS/BDS, especially under conditions of poor observation; 3) Compared with Helmert variance component estimation, it is more appropriate and efficient to assign the weights of different types of observations using equivalent weight ratio. Finally, the ionospheric influence on velocity estimation with single-frequency observations can reach several $\mathrm{mm} / \mathrm{s}$; this influence can be significantly mitigated by using ionosphere-free combination observations.
\end{abstract}

\section{KEYWORDS}
1. GPS
2. BDS.
3. Velocity Estimation.
4. Stand-alone receiver.

Submitted: 2 August 2015. Accepted: 23 November 2015. First published online: 23 December 2015.

1. INTRODUCTION. Although many researchers nowadays mostly focus on Global Navigation Satellite System (GNSS) positioning, navigation and timing technique (e.g. Montenbruck et al., 2014; Li et al., 2015a), velocity estimation still plays an important role in many fields, such as airborne gravimetry, precision agriculture, Rendezvous Docking of aircraft, etc. High-precision velocity estimation using a stand-alone receiver from Global Position System (GPS) broadcast ephemeris has been introduced in many studies (Hebert et al., 1997; Bruton et al., 1999; Serrano et al., 2004a; Van Graas and Soloviev, 2004; Ding and Wang, 2011; Wang and Xu, 2011; Zhang, 2007). The results show that the accuracy of velocity estimation with 
carrier-phase-derived Doppler in a static mode can reach a few $\mathrm{mm} / \mathrm{s}$ and a few $\mathrm{cm} / \mathrm{s}$ in a kinematic mode.

The Chinese BeiDou Navigation Satellite System (BDS) is now providing continuous Positioning, Navigation and Timing (PNT) services to the Asian-Pacific area. By the end of 2014, 16 BDS satellites were launched, which formed a regional positioning system. 14 satellites are in operation, including five Geosynchronous Orbit (GEO) satellites, five Inclined Geosynchronous Orbit (IGSO) satellites, and four Medium Earth Orbit (MEO) satellites (Li et al., 2015b). China plans to launch upgraded satellites and expand its regional BDS to global coverage by 2020 (Yang et al., 2014), so it is necessary and important to evaluate the performance of velocity estimation with BDS satellites.

Generally there are two ways to obtain satellites' velocity: the position-derivation method and the analytic method (by differentiating the position formulae). We compare the results of both methods with the velocity estimation calculated from precise ephemeris using first-order central difference of a Taylor series approximation and find that both methods deliver better than $1 \mathrm{~mm} / \mathrm{s}$ velocity estimation from GPS broadcast ephemeris (Zhang et al., 2006; Serrano et al., 2004a). Nevertheless, we still need to investigate whether these methods can achieve the same performance in BDS. Since the algorithm for IGSO/MEO is the same as GPS, here we just focus on the GEO satellite velocity determination.

As we know, troposphere delay and ionosphere delay are the main error sources in the velocity estimation. Some scholars consider these two errors were highly time correlated and could be mitigated over a short time interval (less than or equal to two seconds) (Ding and Wang, 2011; Serrano et al., 2004a; Zhang and Li, 2012; Li et al., 2014), thus only single-frequency observations are used. However, the ionosphere can be very active and the slant ionospheric delay at lower elevation angle may change significantly, which could decrease the accuracy of velocity estimation. To eliminate this influence, ionosphere-free combination observation (LC) was employed (Van and Soloviev, 2004; Kennedy, 2003; Ge et al., 2008; Li and Zhang, 2012). So far, no results of comparison of these two methods have been published to our knowledge.

Additionally, for a single navigation system, both precision and reliability will deteriorate rapidly in certain environments such as in urban canyons or in mountainous regions where there are insufficient visible satellites. However, this shortage can be compensated by using combined GNSS constellations, which could significantly improve the geometry of observed satellites ( $\mathrm{Li}$ et al., 2015b), and thus improve the precision of velocity estimation as well as its reliability and availability. For multi-systems data processing, precise weighting of the observations from different systems is very important because the measurements of different systems have different noise level and residual errors. To find the proper weights of observations, the Helmert Variance Component Estimation (HVCE) has been widely used (Cai et al., 2014; Koch, 1999). However, this requires a longer time to iterate, high redundant observations, and is not available for real-time application. In order to enhance calculation efficiency, an a priori weight ratio method has more potential.

This paper assesses the performance of velocity estimation using single BDS and combined GPS/BDS in both static and kinematic modes. A poor observation environment will be simulated in static test. Before velocity estimation, the algorithms for realtime BDS GEO satellite Earth-Centred-Earth-Fixed (ECEF) velocity estimation will be 
presented, then compared with a position-derivation method. The accuracy of satellite position estimation and clock drift estimation from BDS broadcast ephemeris will also be analysed. The results using both single- and dual-frequency are shown in order to investigate the influence of ionospheric error on velocity estimation. Finally a comparison of HVCE and an a priori weight ratio method will be demonstrated.

2. GPS/BDS VELOCITY ESTIMATION PROCEDURES. Velocity can be obtained by carrier-phase-derived Doppler (Kennedy et al., 2003; Serrano, 2004b). Considering the observation equation for BDS is similar to that of GPS, only the combined GPS/BDS observation equation is given here:

$$
\begin{gathered}
\dot{\varphi}_{u}^{s}=e_{u}^{s} \cdot\left(\dot{\overrightarrow{\mathrm{r}}}^{s}-\dot{\overrightarrow{\mathrm{r}}}_{u}\right)+c \cdot \dot{t}_{G, u}+c \cdot \dot{\mathrm{t}}_{\mathrm{B}, u}-c \cdot \dot{t}^{s}+\dot{T}_{u}^{s}+\dot{I}_{u}^{s}+\varepsilon \\
e_{u}^{s}=\frac{\overrightarrow{\mathrm{r}}^{s}-\overrightarrow{\mathrm{r}}_{u}}{\rho_{u}} \\
\rho_{u}=\left|\vec{r}^{s}+c \cdot \dot{t}^{s}-\left(\overrightarrow{\mathrm{r}}_{u}+c \cdot \dot{t}_{u}\right)\right|
\end{gathered}
$$

$\dot{\varphi}$ represents the Doppler measurements, $e_{u}^{s}$ the directional cosine, $\rho_{u}$ the geometric distance between receiver $\mathrm{u}$ and satellite $\mathrm{s}, \dot{\overrightarrow{\mathrm{r}}}^{s}$ the satellite velocity vector, $\dot{\overrightarrow{\mathrm{r}}}_{u}$ the receiver velocity vectors, c the speed of light in a vacuum, $\dot{t}_{u}\left(\dot{t}_{G, u}\right.$ and $\left.\dot{t}_{\mathrm{B}, u}\right)$ the receiver clock drift parameters for GPS and BDS, respectively, $t^{s}$ the satellite clock drift (Zhang et al., 2011), $\dot{I}, \dot{T}$ the ionospheric and tropospheric delay rate respectively, $\varepsilon$ the measurements noise.

To reduce the effect of tropospheric delay and ionospheric delay in the observation, the Saastamoinen model (Saastamoinen, 1973) and the ionosphere-free combination observation (LC) (Ge et al., 2008) are employed. As to the stochastic model, we assume that the noise is dependent on elevation, so the sine function of the elevation angle is employed (King and Bock, 1999). The cut-off angle is set to $15^{\circ}$ if there is no special introduction. Since GPS and BDS carrier phase observations have the same level of precision (Yang et al., 2014; Li et al., 2015a), we adopt Equivalent Weight Ratio (EWR) in the process of the combined observations and assume the observations are independent. When all errors are modelled or negligible, we can describe the unknown parameters as $X_{v}=\left[\dot{\overrightarrow{\mathrm{r}}}_{u} \dot{t}_{G, u} \dot{t}_{B, u}\right]$ in the Least Square adjustment.

\section{THE BDS BROADCAST EPHEMERIS ANALYSIS. According to Equation} (1), it can be seen that high-precision velocity estimation not only depends on the accuracy of the measurements, but also depends on the accuracy of satellite position, velocity, clock drift calculated from the broadcast ephemeris and the accuracy of Single Point Positioning (SPP). These effects have been analysed for GPS (Zhang, 2007). However, only a few research efforts focus on these effects for BDS. Yang et al. (2014) show that the accuracy of SPP in BDS is better than $10 \mathrm{~m}$ in the Asia-Pacific region, which is in the same level of magnitude to that of GPS. In this consideration, the positioning error can be ignored. Thus, it is meaningful to make a comprehensive analysis of BDS broadcast ephemeris.

An algorithm for BDS satellite ECEF position and satellite clock drift from the broadcast ephemeris is presented in the BeiDou-ICD-2·0-2013 (CSNO, 2013). 
Satellite velocity can be obtained from position differential and closed-form formulae. Since the algorithm for IGSO/MEO is the same as that of GPS, we just give the GEO satellite velocity closed-form formula according to the position algorithm.

GEO satellite position in ECEF can be expressed as (CSNO, 2013):

$$
\left[\begin{array}{c}
X \\
Y \\
Z
\end{array}\right]_{E C E F}=R_{Z}\left(\omega t_{k}\right) R_{X}\left(-5^{\circ}\right) R\left[\begin{array}{l}
x \\
y \\
0
\end{array}\right]
$$

where:

$$
\begin{aligned}
R & =\left[\begin{array}{ccc}
\cos L & -\sin L \cos I & \sin L \sin I \\
\sin L & \cos L \cos I & -\cos L \sin I \\
0 & \sin I & \cos I
\end{array}\right], R_{Z}\left(\omega t_{k}\right) \\
& =\left[\begin{array}{ccc}
\cos \left(\omega t_{k}\right) & \sin \left(\omega t_{k}\right) & 0 \\
-\sin \left(\omega t_{k}\right) & \cos \left(\omega t_{k}\right) & 0 \\
0 & 0 & 1
\end{array}\right], R_{X}\left(-5^{\circ}\right)=\left[\begin{array}{ccc}
1 & 0 & 0 \\
0 & \cos 5^{\circ} & \sin 5^{\circ} \\
0 & -\sin 5^{\circ} & \cos 5^{\circ}
\end{array}\right]
\end{aligned}
$$

$(\mathrm{x}, \mathrm{y}, 0)$ denotes the satellite position in the natural orbit plane system, $\mathrm{L}$ the longitude of the ascending node, I the orbit inclination, $\omega$ the earth rotation parameter, $t_{k}$ the interval between time of observation and reference ephemeris.

The satellite velocity vector $(\dot{X}, \dot{Y}, \dot{Z})$ can be obtained by taking the derivative of the satellite position:

$$
\begin{gathered}
{\left[\begin{array}{c}
\dot{X} \\
\dot{Y} \\
\dot{Z}
\end{array}\right]=\dot{R}_{Z}\left(\omega t_{k}\right) R_{X}\left(-5^{\circ}\right) R\left[\begin{array}{l}
x \\
y \\
0
\end{array}\right]+R_{Z}\left(\omega t_{k}\right) R_{X}\left(-5^{\circ}\right) \dot{R}\left[\begin{array}{l}
x \\
y \\
0
\end{array}\right]} \\
+R_{Z}\left(\omega t_{k}\right) R_{X}\left(-5^{\circ}\right) R\left[\begin{array}{l}
\dot{x} \\
\dot{y} \\
0
\end{array}\right] \\
\dot{R}_{Z}\left(\omega t_{k}\right)=\left[\begin{array}{ccc}
-\omega \sin \left(\omega t_{k}\right) & \omega \cos \left(\omega t_{k}\right) & 0 \\
-\omega \cos \left(\omega t_{k}\right) & -\omega \sin \left(\omega t_{k}\right) & 0 \\
0 & 0 & 0
\end{array}\right], \\
\dot{R}=\left[\begin{array}{ccc}
-\dot{L} \sin L & -\dot{L} \cos L \cos I+\dot{I} \sin L \sin I & \dot{L} \cos L \sin I+\dot{I} \sin L \cos I \\
\dot{L} \cos L & -\dot{L} \sin L \cos I-\dot{I} \cos L \sin I & \dot{L} \sin L \sin I-\dot{I} \cos L \cos I \\
0 & \dot{I} \cos I & -\dot{I} \sin I
\end{array}\right]
\end{gathered}
$$

where the dot represents the first derivative with respect to time.

Figure 1 shows that the residuals of the satellite 3D velocity for C03(GEO), C06 (IGSO), C14(MEO) from the differentiation method are better than the closed-form formula: although manifesting systematic bias, its precision is better than $1 \mathrm{~mm} / \mathrm{s}$. The main explanations are as follow: (1) Orbit errors are absorbed into velocity parameters when we use the analytic method directly. (2) Due to the high stability of the satellite orbit, the satellite position error even up to $10 \mathrm{~m}$ can be suppressed by differentiation. Figure 2 depicts the accuracy of satellite positions and clock drifts calculated from BDS broadcast ephemeris. Obviously, it can meet the demands of high-precision velocity estimation. 


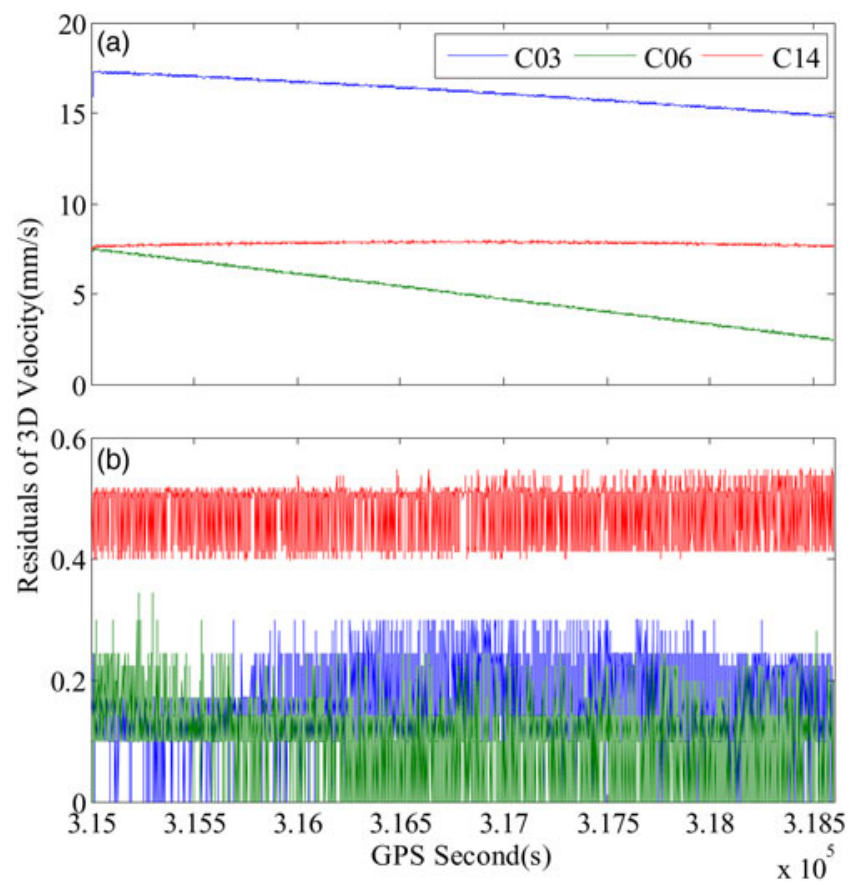

Figure 1. Residuals of satellite velocity obtained from the closed-form formula (a) and positionderivation (b) compared with the velocity obtained from the SP3 precise ephemeris using firstorder central difference of a Taylor series approximation $(\mathrm{PRN}=\mathrm{C} 03 / \mathrm{C} 06 / \mathrm{C} 14,15: 30: 00 \sim 16: 30: 00$, $10 / 01 / 2014)$.
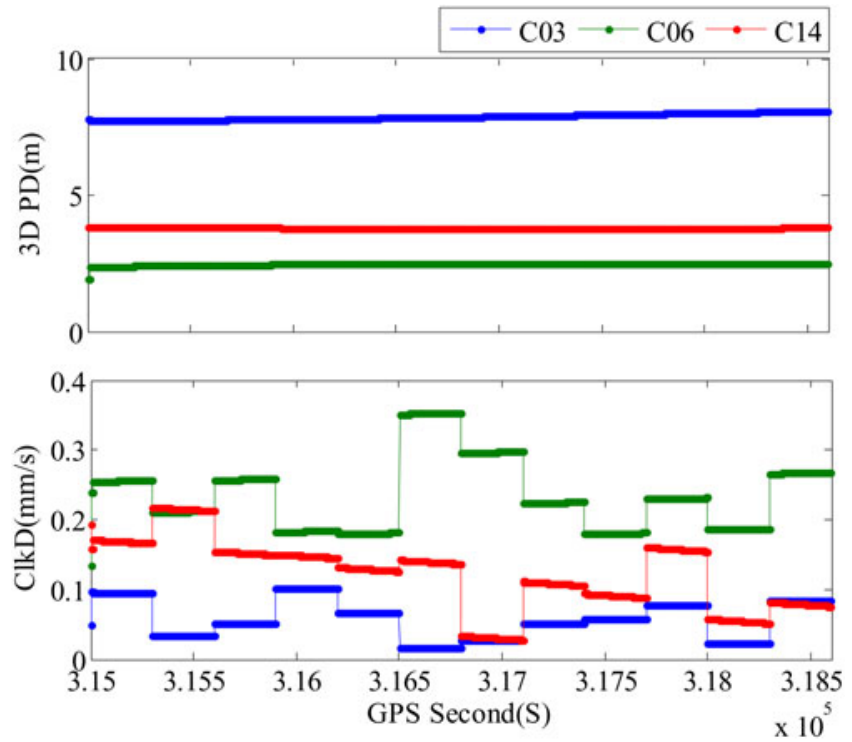

Figure 2. Proof that the satellite position and clock drift calculated by using the broadcast ephemeris is sufficiently accurate in comparison with SP3 precise ephemeris ( $\mathrm{PRN}=\mathrm{C} 03 / \mathrm{C06} /$ C14,15:30:00 16:30:00, 10/01/2014). 


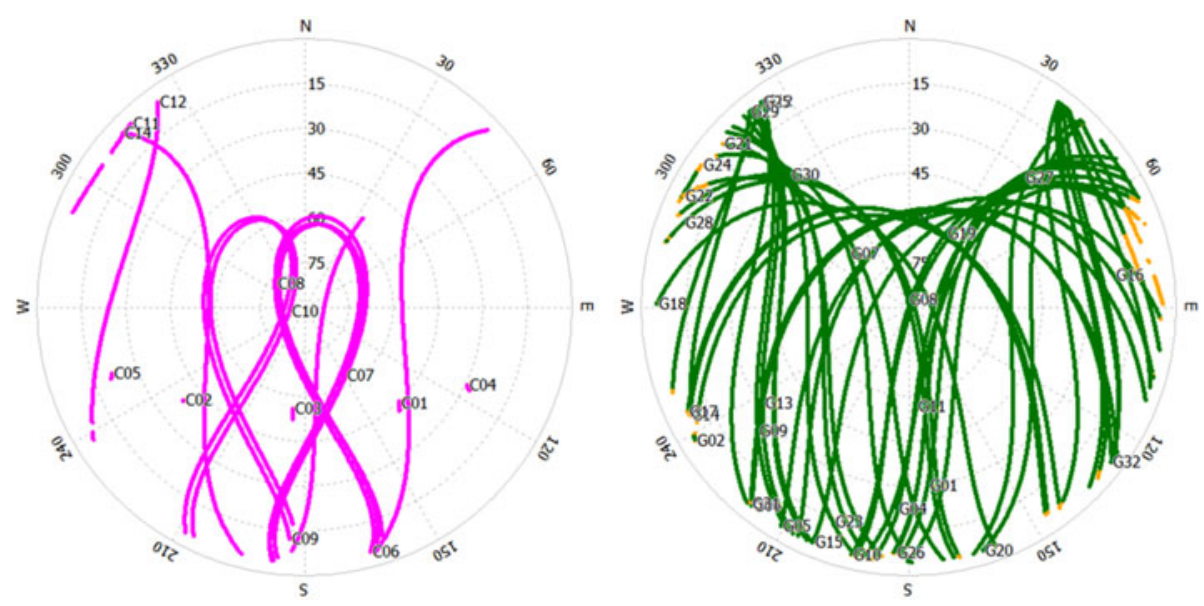

Figure 3. Sky plots of BDS (left) and GPS (right) satellites of station WHCD. The purple lines represent observations with $\mathrm{B} 1 / \mathrm{B} 2$ frequencies, the green lines represent observations with L1/L2 frequencies, and the yellow lines represent observations with L1 frequency only.

4. STATIC TEST. In order to assess the performance of stand-alone velocity estimation for GPS/BDS comprehensively, three experiment schemes were designed: scheme1 single-frequency (SF) (B1, L1), scheme2, LC with cut-off angle $15^{\circ}, 30^{\circ}$ and $45^{\circ}$, scheme 3 LC (B1/B2, L1/L2) with HVCE method. Dual-frequency GPS/ BDS data sets from eight stations (WHCD, WHDH, WHEZ, WHHN, WHHP, WHKC, WHQJ, WHXT) at the Wuhan CORS on 1 October 2014 were collected with sampling interval of $1 \mathrm{~s}$. Since true velocity is zero, the test results would help to reveal the sources and characteristics of estimation error.

Take the station WHCD as an example, the sky plots of GPS and BDS satellites are shown in Figure 3, and the corresponding dilution of precision in horizontal (HDOP) and vertical (VDOP) directions are given in Figure 4. It can be seen that, compared with a GPS-only system, the mean HDOP, VDOP of integrated GPS/BDS increased nearly $30 \%$ and $33 \%$. This means the geometry is significantly improved in the integrated GPS/BDS. As for BDS, the HDOP and VDOP are worse than GPS because the satellites distribution is incomplete.

The ionospheric error is a vital error source in data processing ( $\mathrm{Li}$ et al., 2013). Comparing Figure 5 (scheme1) and Figure 7 (scheme2), we can see that many fluctuations occur in the three components of velocity estimation for GPS, especially during the GPS Second 280000 294000 (in the red rectangle box), while for BDS, there is only a small difference. The reason is that the ionospheric delay change rates along the signal transmitting path of GEO satellites are slower than that of IGSO, MEO satellites. This is due to the fact that GEO satellites are stationary with respect to the earth, thus the impact of ionospheric delay on velocity estimation in BDS is less than that of GPS. Besides, LC combinations are preferred in active ionosphere environments, such as ionospheric disturbance.

The precision statistics based on the results of these eight stations are illustrated in Figure 6. Obviously, the accuracy of North and East components are better than the Up component. The Root Mean Square (RMS) and mean value of BDS are already 

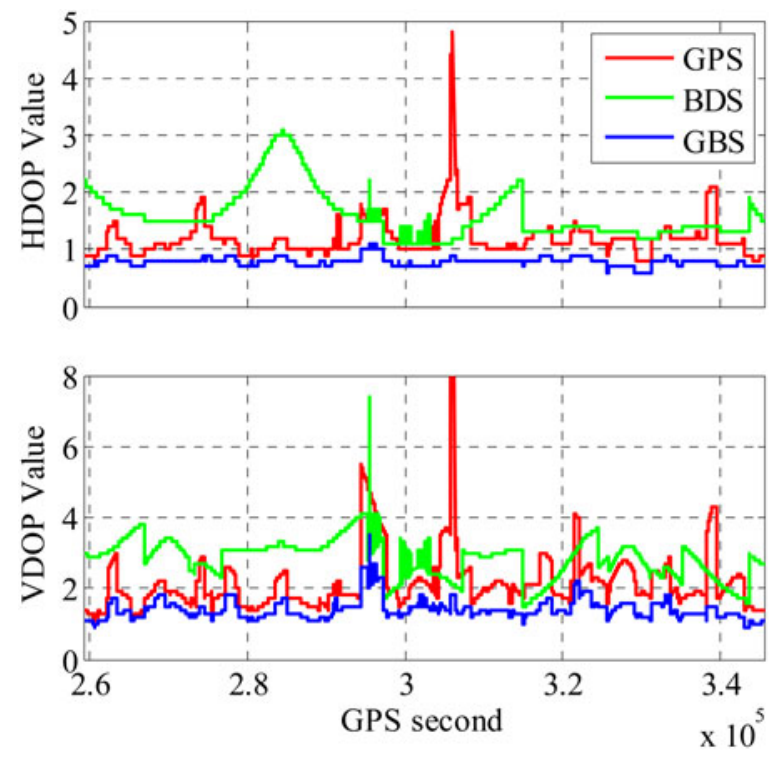

Figure 4. HDOP, VDOP of GPS, BDS and GPS/BDS (station: WHDH).
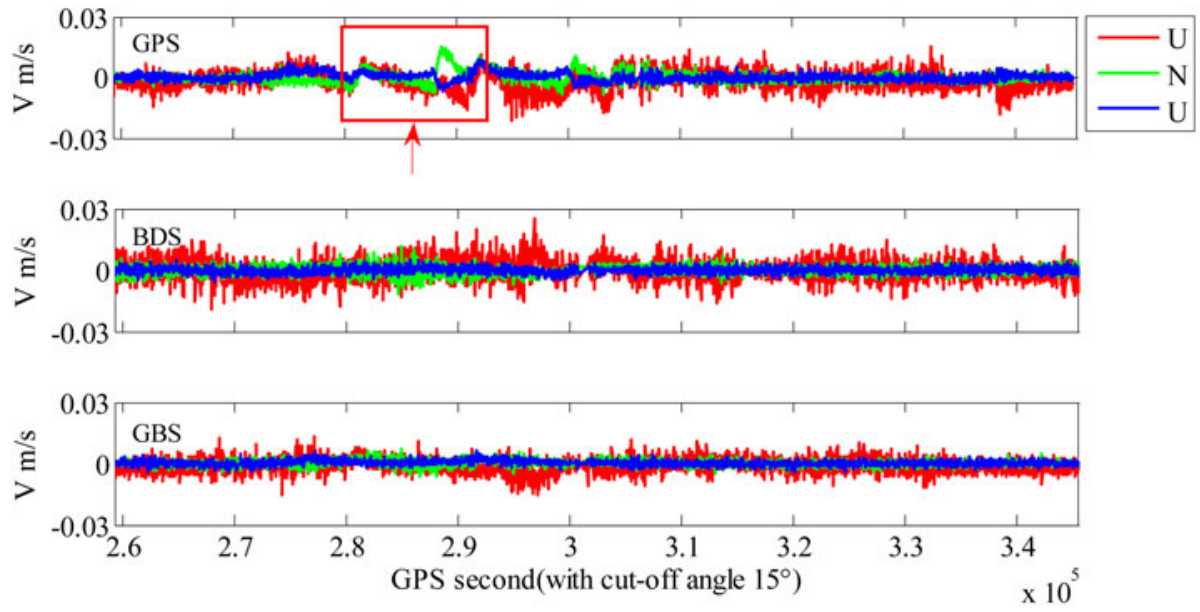

Figure 5. Scheme1 velocity estimation using SF for East/North/Up components (station: WHCD).

comparable to GPS, where the mean value is nearly $0 \cdot 0$ in the horizontal component and $1 \mathrm{~mm} / \mathrm{s}$ in the vertical component, the RMS is better than $2 \mathrm{~mm} / \mathrm{s}$ in the horizontal and within $5 \mathrm{~mm} / \mathrm{s}$ in the upward component. Compared to a GPS-only system, the RMSs of the combined system improves 14\%, 23\% and 24\% in East/North/Up components respectively, and the advantage will be more remarkable in challenging environments.

To simulate poor observation conditions, we design scheme 2 and the results are depicted in Figure 7, Figure 8, and Figure 9. From Table 1, we can see that with 

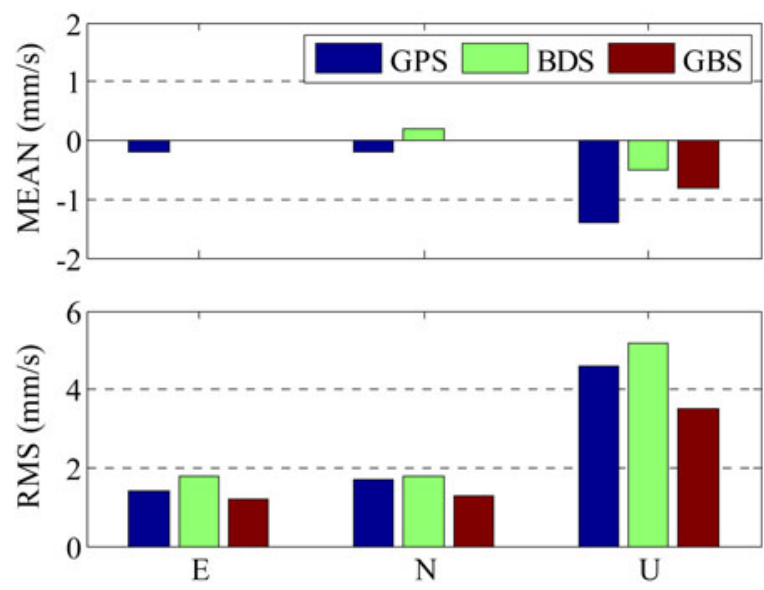

Figure 6. The precision statistics of velocity estimation for GPS, BDS and GPS/BDS using LC from 8 stations.
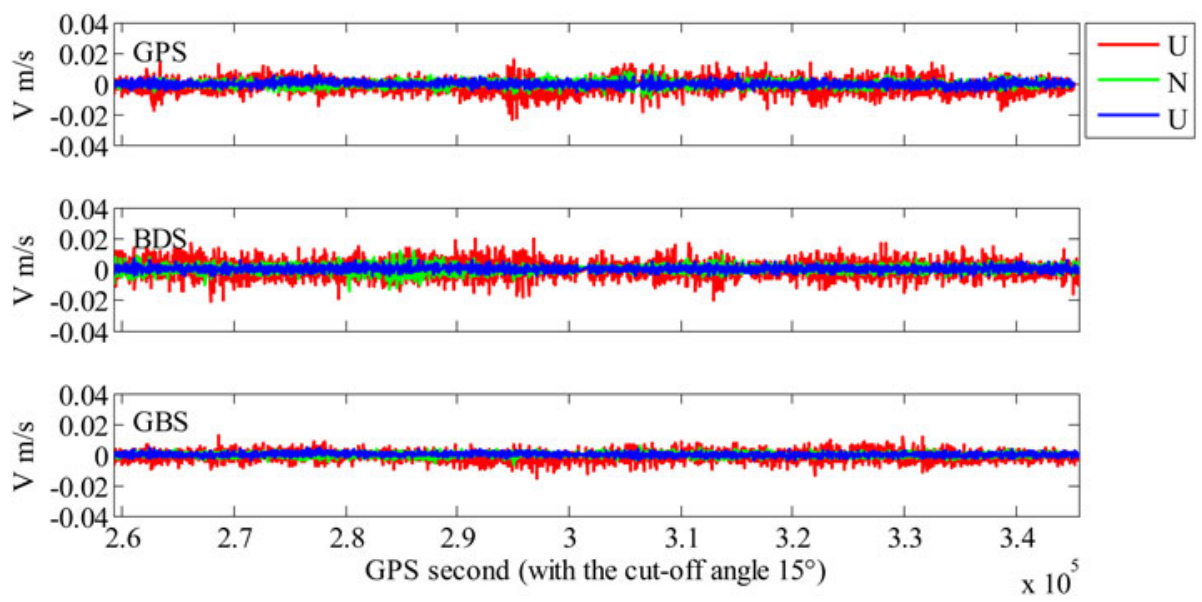

Figure 7. Scheme2 Velocity estimation using LC for East/North/Up components with cut-off angle $15^{\circ}$ (station: WHCD).

cut-off angle increasing, the number of visible satellites decreases rapidly, and the geometry strength becomes weaker, consequently, the accuracy as well as reliability of velocity estimation decreases. Furthermore, for GPS/BDS, the Percentage of Valid Epochs (PVE) which can be used in the calculation still reaches $97 \%$ during the test, which quite outnumbers that of the single system.

Table 2 illustrates the precision statistics of scheme 2 (cut-off angle $15^{\circ}$ ) and scheme 3 , in respect of precision statistics, they are mostly consistent. Furthermore, the program running time improves almost $25 \%$ by the EWR method. To further verify the applicability and reliability of EWR, the variance ratios of GPS/BDS from HVCE are calculated. These values are ranges from $0 \cdot 7$ to $1 \cdot 5$. Their mean value is $1 \cdot 13$, and RMS is $1 \cdot 18$. These show the EWR is well supported. 

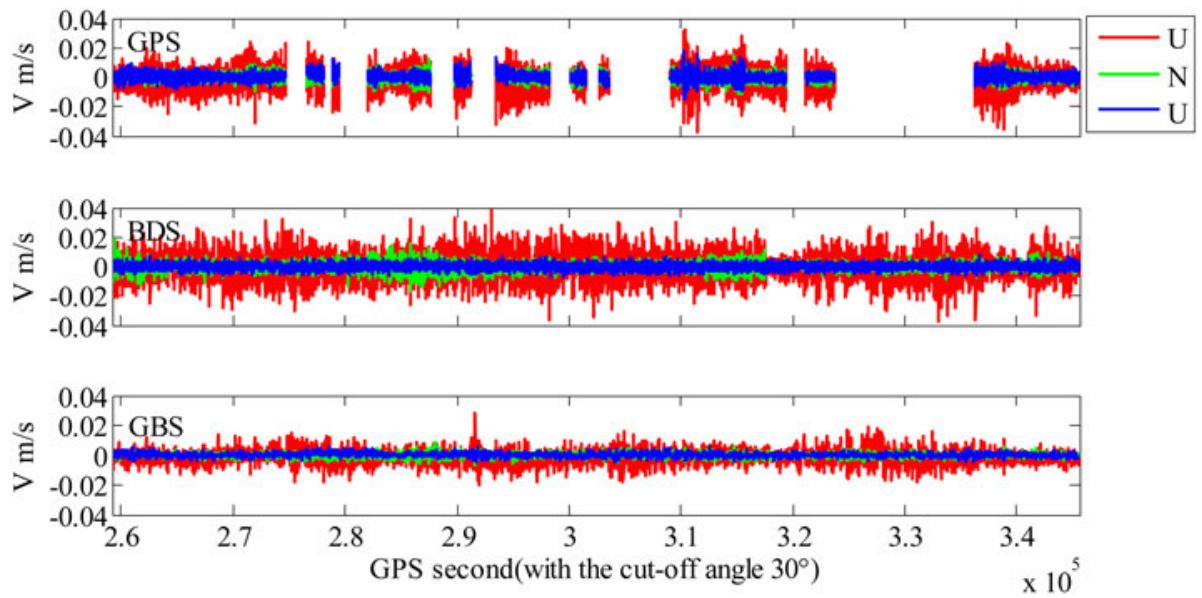

Figure 8. Scheme2 Velocity estimation using LC for East/North/Up components with cut-off angle $30^{\circ}$ (station: WHCD).
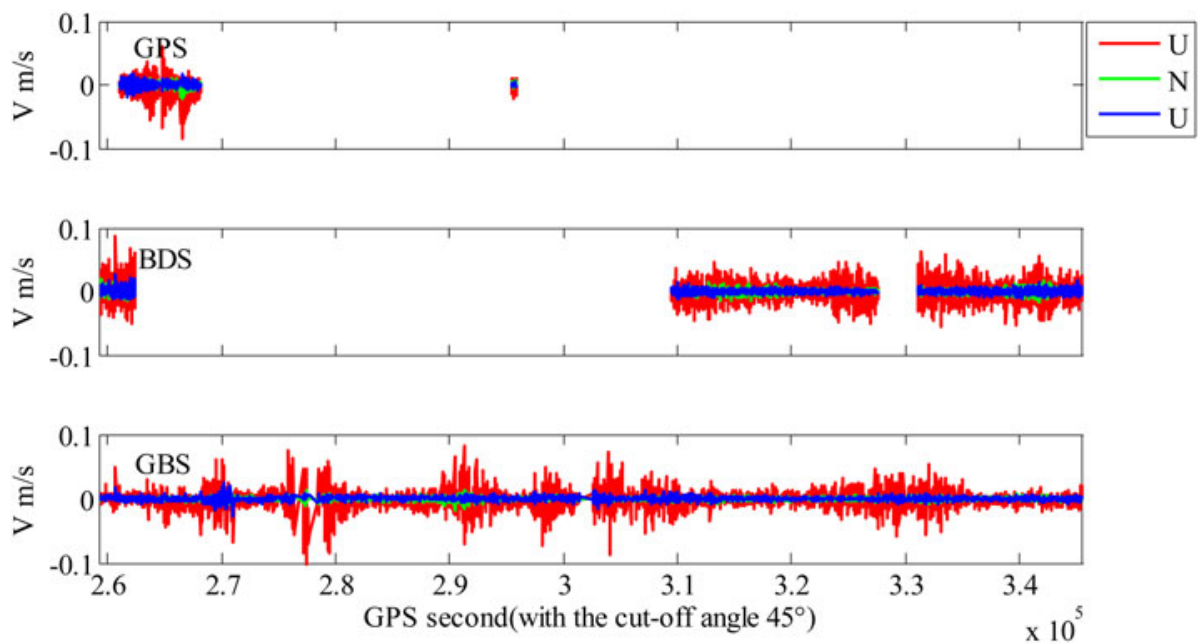

Figure 9. Scheme2 Velocity estimation using LC for East/North/Up components with the cut-off angle $45^{\circ}$ (station: WHCD).

Table 1. Percentage of valid epochs and corresponding visible satellite number.

\begin{tabular}{|c|c|c|c|c|c|c|}
\hline \multirow[t]{2}{*}{ Cut-off elevation( $\left(^{\circ}\right)$} & \multicolumn{2}{|l|}{ GPS } & \multicolumn{2}{|l|}{ BDS } & \multicolumn{2}{|c|}{ GPS/BDS } \\
\hline & PVE & visible number & PVE & visible number & PVE & visible number \\
\hline 15 & $99 \%$ & $5 \sim 12$ & $99 \%$ & $7 \sim 11$ & $99 \%$ & $11 \sim 20$ \\
\hline 30 & $62 \%$ & $3 \sim 9$ & $99 \%$ & $5 \sim 9$ & $99 \%$ & $9 \sim 16$ \\
\hline 45 & $8 \%$ & $<6$ & $41 \%$ & $<7$ & $97 \%$ & $5 \sim 11$ \\
\hline
\end{tabular}


Table 2. Statistic and program running time of stand-alone velocity estimation using HVCE and EWR.

\begin{tabular}{lcccccc}
\hline & & rms (cm/s) & mean (cm/s) & max bias $(\mathbf{c m} / \mathbf{s})$ & $\min$ bias $(\mathbf{c m} / \mathbf{s})$ & Program running time(s) \\
\hline EWR & $\mathrm{E}$ & $0 \cdot 1$ & $0 \cdot 0$ & $0 \cdot 7$ & $-0 \cdot 8$ & $173 \cdot 464$ \\
& $\mathrm{~N}$ & $0 \cdot 2$ & $0 \cdot 0$ & $0 \cdot 7$ & $-1 \cdot 1$ & \\
& $\mathrm{U}$ & $0 \cdot 4$ & $-0 \cdot 1$ & $1 \cdot 7$ & $-2 \cdot 2$ & \\
HVCE & $\mathrm{E}$ & $0 \cdot 1$ & $0 \cdot 0$ & $0 \cdot 8$ & $-0 \cdot 6$ & $216 \cdot 675$ \\
& $\mathrm{~N}$ & $0 \cdot 2$ & $0 \cdot 0$ & $1 \cdot 1$ & $-1 \cdot 2$ & \\
& $\mathrm{U}$ & $0 \cdot 4$ & $-0 \cdot 1$ & $2 \cdot 7$ & $-2 \cdot 7$ & \\
\hline
\end{tabular}

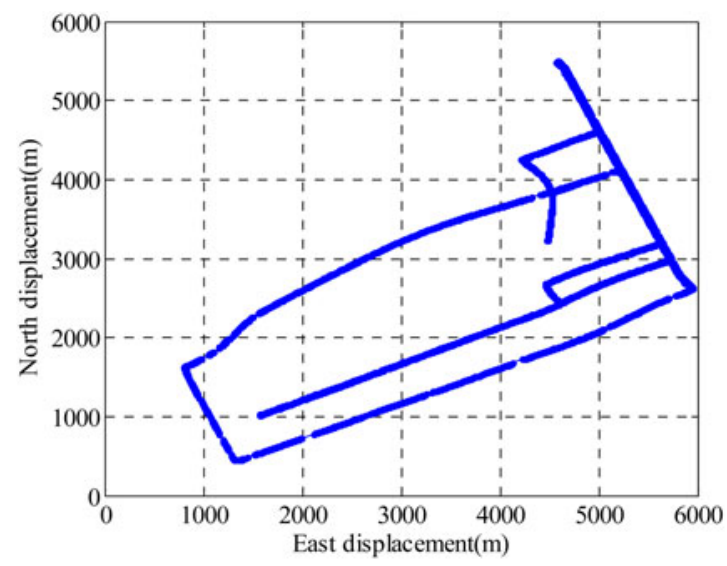

Figure 10. The car's ground track.

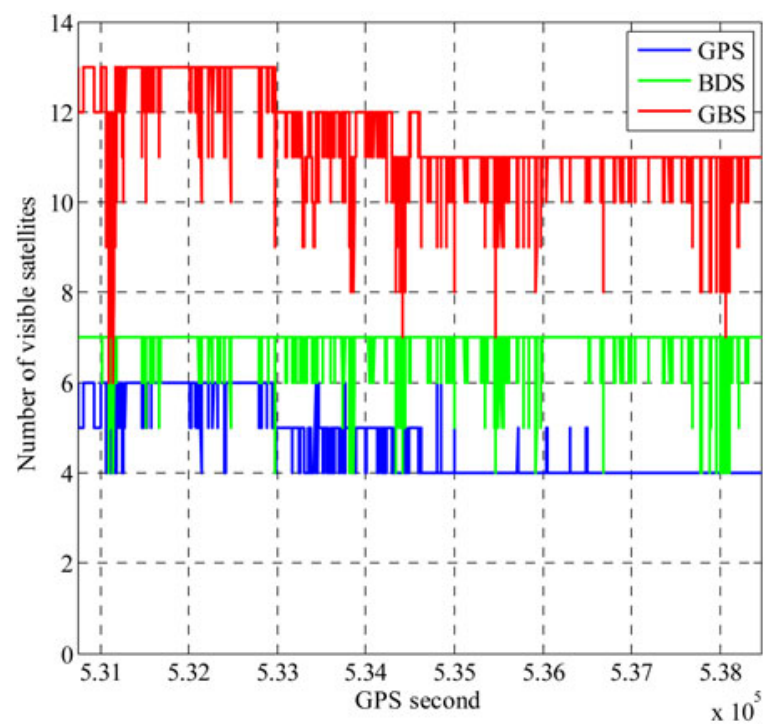

Figure 11. Number of the tracked satellites during the test. 

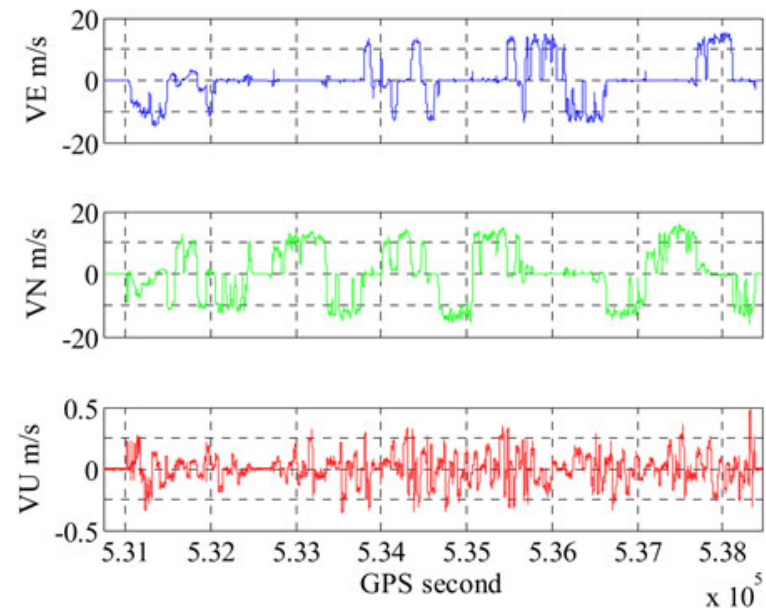

Figure 12. Velocity obtained from DGPS with first-order central difference of a Taylor series approximation. These three panels illustrate East, North and Up components of the velocity.

(a)
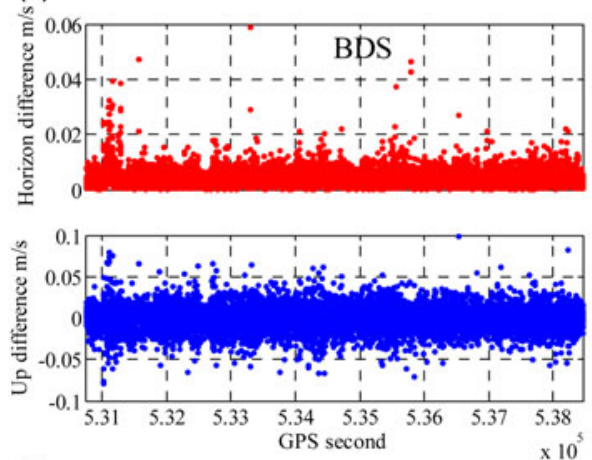

(c)
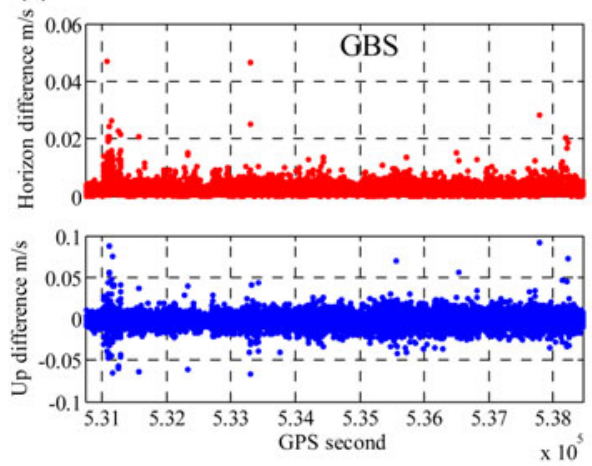

(b)
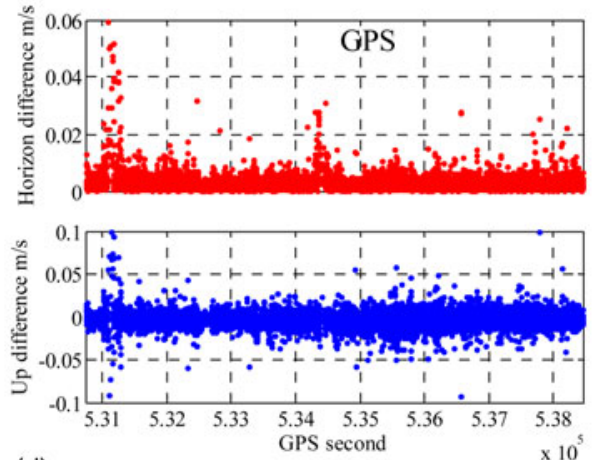

(d)
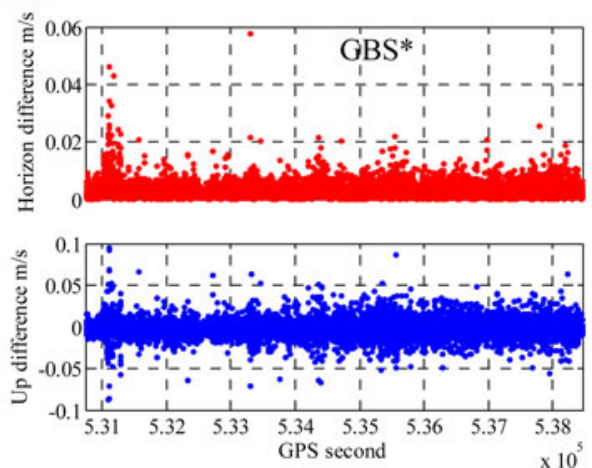

Figure 13 . The 3D velocity difference between the stand alone velocity solution and the reference velocity. 
Table 3. Accuracy and valid epochs statistics of stand-alone velocity.

\begin{tabular}{|c|c|c|c|c|c|}
\hline & & $\mathrm{rms}(\mathrm{cm} / \mathrm{s})$ & mean $(\mathrm{cm} / \mathrm{s})$ & $3 \mathrm{D} \mathrm{rms}(\mathrm{cm} / \mathrm{s})$ & Number of valid epoch \\
\hline \multirow[t]{3}{*}{ GPS } & $\mathrm{E}$ & 0.5 & $0 \cdot 0$ & $1 \cdot 6$ & 6945 \\
\hline & $\mathrm{N}$ & 0.6 & $0 \cdot 0$ & & \\
\hline & $\mathrm{U}$ & $1 \cdot 4$ & $0 \cdot 2$ & & \\
\hline \multirow[t]{3}{*}{ BDS } & E & $0 \cdot 4$ & $0 \cdot 0$ & 1.8 & 7150 \\
\hline & $\mathrm{N}$ & $0 \cdot 4$ & $0 \cdot 0$ & & \\
\hline & $\mathrm{U}$ & $1 \cdot 7$ & $0 \cdot 0$ & & \\
\hline \multirow[t]{3}{*}{ GBS } & E & $0 \cdot 3$ & $0 \cdot 0$ & $1 \cdot 0$ & 7167 \\
\hline & $\mathrm{N}$ & $0 \cdot 2$ & $0 \cdot 0$ & & \\
\hline & $\mathrm{U}$ & 0.9 & $0 \cdot 1$ & & \\
\hline \multirow{3}{*}{ GBS* } & $\mathrm{E}$ & $0 \cdot 4$ & $0 \cdot 0$ & 1.5 & 7167 \\
\hline & $\mathrm{N}$ & 0.7 & $0 \cdot 0$ & & \\
\hline & $\mathrm{U}$ & $1 \cdot 3$ & $0 \cdot 1$ & & \\
\hline
\end{tabular}

5. KINEMATIC TEST. The implemented kinematic test was carried out in Jiangxia District, Wuhan, on 7 June 2014. Dual frequency carrier phase measurements were collected using a South-GNSS-S82C receiver with a sampling interval of 1s. The rover receiver was mounted on a car and its trajectory is shown in Figure 10. The number of satellites tracked is shown in Figure 11. The base receiver was installed on an open site. Since it is very difficult to obtain true velocity in a kinematic test, in our case, we use the reference velocity that was derived through numerical differentiation of the receiver positions that were processed by post processing Differential GPS techniques (DGPS) (Ding and Wang, 2011) with a commercial software tool, i.e. GrafNav, shown in Figure 12.

Figure 13 depicts 3D velocity difference between the stand-alone velocity solution and that of DGPS, wherein the results calculated by HVCE are marked with '*'. It can be seen that the residual noise of BDS is at the same level of magnitude as that of GPS in the horizontal component but worse in the Up component, and for GPS/BDS, the residual noise is the least. From Table 3, we can see that the number of valid epochs for GPS is less than BDS and GPS/BDS. The reason is that some GPS satellites signal were obscured during the test, while there were five BDS satellites at high-elevation angles above $30^{\circ}$. Although HVCE is a rigorous weighting approach, which requires high redundant observations, these could not be always satisfied in poor observation conditions, thus resulting in unreliable weight solutions and low reliability of velocity estimation. The EWR works quite well, considering its mean value of residuals shown above appear to be unbiased, its RMS of horizontal component is below $0.3 \mathrm{~cm} / \mathrm{s}$ and its RMS of the upward component is nearly $1 \mathrm{~cm} / \mathrm{s}$. Above all the results of the kinematic test agree with the former static test very well.

6. CONCLUSIONS. In this paper, we evaluated the performance of velocity estimation of the BDS as well as the integrated GPS/BDS. The conclusions are as follows:

Compared with position-derivation method from precise ephemeris SP3, the BDS satellite velocity using position-derivation method whose accuracy is above $1 \mathrm{~mm} / \mathrm{s}$ is better than closed-form formulae from broadcast ephemeris. In addition it simplifies the velocity transformation procedure, and provides a good alternative. Both the 
satellite position and clock drift obtained from BDS broadcast ephemeris meet the demands of high precision velocity estimation.

When the satellite geometry distribution is similar, the results of BDS are comparable to GPS, and its stability and accuracy noticeably improve in the integrated GPS/ BDS, especially under poor observation conditions.

Compared with LC, the SF can be influenced by ionosphere delay, which can cause errors as much as $1 \sim 2 \mathrm{~cm} / \mathrm{s}$, thus we prefer LC in practice.

It is appropriate and applicable to use a prior weight, i.e. EWR in processing the GPS/BDS combined observations, whose performance is at the same level of magnitude or even better than that of HVCE which is limited by redundant observations, and because there is no need of iteration, the EWR is more efficient.

\section{ACKNOWLEDGEMENTS}

This work is supported by the Natural Science Foundation for Distinguished Young Scholar of Hubei Province (No: 2015CFA039) and the Spark Program of Earthquake Sciences (No. XH16053).

\section{REFERENCES}

Bruton, A. M., Glennie, C. L. and Schwarz, K. P. (1999). Differentiation for high-precision GPS velocity and acceleration determination. GPS solutions, 2(4), 7-21.

Cai, C., Pan, L. and Gao, Y. (2014). Precise Weighting Approach with Application to Combined L1/B1 GPS/ BeiDou Positioning. The Journal of Navigation, 67(05), 911-925.

CSNO. (2013). BeiDou Navigation Satellite System Signal in Space Interface Control Document Open Service Signal (Version 2.0) (Beidou-ICD-2·0-2013). Available: http://en.beidou.gov.cn/

Ding, W. and Wang, J. (2011). Precise velocity estimation with a stand-alone GPS receiver. Journal of Navigation, 64(02), 311-325.

Ge, M., Gendt, G., Rothacher, M., Shi, C. and Liu, J. (2008). Resolution of GPS carrier-phase ambiguities in precise point positioning (PPP) with daily observations. Journal of Geodesy, 82(7), 389-399.

Hebert, J., Keith, J., Ryan, S., Szarmes, M., Lachapelle, G. and Cannon, M. E. (1997). DGPS kinematic carrier phase signal simulation analysis for precise aircraft velocity determination. Proceedings of Annual Meeting of the Institute of Navigation, 44(2), 231-245.

Kennedy, S.L. (2003). Precise acceleration determination from carrier-phase measure- ments. Navigation Journal of the Institute of Navigation, 50, 9-19.

Koch, K.R. (1999). Parameter Estimation and Hypothesis Testing in Linear Models (Second, Updated and Enlarged Edition). Berlin: Springer.

King, R.W. and Bock, Y. (1999). Documentation for the GAMIT GPS analysis software. Mass. Inst. of Technol., Cambridge Mass.

Li, X. and Zhang, X. (2012). Improving the Estimation of Uncalibrated Fractional Phase Offsets for PPP Ambiguity Resolution. The Journal of Navigation, 65, 513-529.

Li, X., Ge, M., Zhang, H. and Wickert, J. (2013). A method for improving uncalibrated phase delay estimation and ambiguity-fixing in real-time precise point positioning. Journal of Geodesy, 87(5), 405-416.

$\mathrm{Li}, \mathrm{X}$., Zhang, X. and Guo, F. (2014). Predicting atmospheric delays for rapid ambiguity resolution in precise point positioning. Advance in Space Research. 54(5), 840-850.

Li, X., Zhang, X., Ren, X., Fritsche, M., Wickert, J. and Schuh, H. (2015a). Precise positioning with current multi-constellation Global Navigation Satellite Systems: GPS, GLONASS, Galileo and BeiDou. Sci Rep., $\mathbf{5}, 8328$.

Li, X., Ge, M., Dai, X., Ren, X., Fritsche, M.,Wickert, J. and Schuh, H. (2015b). Accuracy and reliability of multi-GNSS real-time precise positioning: GPS, GLONASS, BeiDou, and Galileo. Journal of Geodesy, 89(6), 607-635. 
Montenbruck, O., Steigenberger, P., Khachikyan, R., Weber, G., Langley, R.B., Mervart, L. and Hugentobler, U. (2014). IGS-MGEX: preparing the ground for multi-constellation GNSS science. Inside GNSS, 9(1), 42-49.

Serrano, L., Kim, D. and Langley, R. B. (2004a). A single GPS receiver as a real-time, accurate velocity and acceleration sensor. Proceedings of the $17^{\text {th }}$ International Technical Meeting of the Satellite Division of The Institute of Navigation (ION GNSS 2004), Long Beach, CA.

Serrano, L., Kim, D., Langley, R. B., Itani, K., and Ueno, M. (2004b). A GPS velocity sensor: how accurate can it be?-a first look. In ION NTM, 875-885.

Saastamoinen, J. (1973). Contributions to the theory of atmospheric refraction[J]. Bulletin Géodésique (19461975), 107(1), 13-34.

Van, Graas. F. and Soloviev, A. (2004). Precise velocity estimation using a stand-alone GPS receiver. Navigation, 51(4), 283-292.

Wang, Q. and Xu, T. (2011). Combining GPS carrier phase and Doppler observations for precise velocity determination. Science China Physics, Mechanics and Astronomy, 54(6), 1022-1028.

Yang, Y., Li, J., Wang, A., Xu, J., He, H., Guo, H., Shen, J and Dai, X. (2014). Preliminary assessment of the navigation and positioning performance of BeiDou regional navigation satellite system. Science China Earth Sciences, 57(1), 144-152.

Zhang, X., and Li, X. (2012). Instantaneous Re-initialization in Real-time Kinematic PPP with Cycle-slips Fixing. GPS solutions, 16(3), 315-327.

Zhang, X., Li, X., Guo, F. (2011). Satellite Clock Estimation at $1 \mathrm{~Hz}$ for Realtime Kinematic PPP applications. GPS solutions, 15(4), 315-324.

Zhang, J., Zhang, K., Grenfell, R. and Deakin, R. (2006). GPS Satellite Velocity and Acceleration Determination using the Broadcast Ephemeris. Journal of Navigation, 59(02), 293-305.

Zhang, J. (2007). Precise velocity and acceleration determination using a standalone GPS receiver in real time, $P h D$ thesis. 